\title{
ACTORES NO ESTATALES Y PROCESOS DE INTEGRACIÓN REGIONAL: LA UNIÓN EUROPEA Y EL SISTEMA DE INTEGRACIÓN CENTROAMERICANO
}

\section{NON STATE ACTORS AND REGIONAL INTEGRATION PROCESSES: THE EUROPEAN UNION AND THE CENTRAL AMERICAN INTEGRATION SYSTEM}

\author{
Kevin Parthenay ${ }^{*}$ \\ Rosa Sánchez Salgado**
}

\begin{abstract}
RESUMEN
Este estudio constituye un análisis comparativo de las interacciones entre actores no estatales (denominados sociedad transnacional en este artículo) y procesos de integración regional en Europa y América Central. En un primer momento, se resalta la correlación existente entre el nivel de desarrollo de las instituciones regionales $y$ el florecimiento de una sociedad transnacional. En segundo lugar, se presentan las relaciones entre actores no estatales e instituciones regionales en el marco de la Unión Europea (UE) y del Sistema de Integración Centroamericano (SICA). A pesar de las importantes diferencias contextuales entre Europa y Centroamérica, en ambos casos, los actores no estatales son percibidos como una fuente de legitimidad para las instituciones regionales. Asimismo, estos actores también son solicitados por sus competencias técnicas. La evidencia empírica proviene del análisis de documentación primaria y secundaria, además de entrevistas semi-directivas de los actores más relevantes.
\end{abstract}

PALABRAS CLAVE: EUROPA * AMÉRICA CENTRAL * INTEGRACIÓN REGIONAL * SOCIEDADES TRANSNACIONALES * ACTORES NO ESTATALES * SOCIEDAD CIVIL

Centro de Estudios e Investigaciones Internacionales (CERI).

kevin.parthenay@sciences-po.org
** Universidad de Amsterdam (UVA), Holanda y Centro de Estudios Europeos (CEE).

R.M.Sanchez@uva.nl 


\section{ABSTRACT}

This study advances a comparative analysis of the interactions between non state actors (in this article also referred to as transnational society) and regional integration processes in Europe and Central America. Firstly, the correlation between the degree of development of regional institutions and the flowering of a transnational society is highlighted. Secondly, the attention is drawn to the current relationships between non state actors and regional institutions in the European Union (EU) and the Central American Integration System (SICA). A particular attention will be paid to civil society participation mechanisms within those regional institutions. In spite of the significant contextual differences between Europe and Central America, in both cases non state actors are viewed as a potential source of political legitimacy for regional institutions, as well as suppliers of technical expertise. Empirical evidence is drawn from the analysis of primary and secondary documents as well as from semidirective interviews to the most relevant actors.

KEYWORDS: EUROPE * CENTRAL AMERICA * REGIONAL INTEGRATION * TRANSNATIONAL CORPORATION * NON STATE ACTORS * CIVIL SOCIETY

\section{INTRODUCCIÓN}

El estudio de la integración europea ha monopolizado la gran mayoría de esfuerzos de conceptualización y teorización de los procesos de integración regional. A pesar de las aspiraciones de algunas teorías de la integración, se resalta la escasez de estudios comparativos, tanto desde el punto de vista teórico como empírico (Schmitter, 1970). Este artículo constituye una primera contribución al desarrollo de una perspectiva comparativa de los procesos de integración considerados como espacios de interacción entre actores sociales. Este esfuerzo comparativo contribuye por tanto a la "deseuropeización" de los estudios académicos sobre la integración regional.

Tal perspectiva comparativa hubiera podido aplicarse a múltiples ámbitos de los procesos de integración regional. La originalidad de este artículo también estriba en que se analiza la interacción entre actores no estatales y procesos de integración regional. La mayoría de estudios empíricos sobre los procesos de integración regional analizan los intercambios económicos o el funcionamiento institucional y solo recientemente, se está empezando a prestar una atención suficiente a los actores sociales (EPPIE, 2007: 13-86).

A continuación, se presenta el marco teórico que fundamenta la comparación de los procesos de integración regional en Europa y Centroamérica. Seguidamente, se trata de determinar la importancia de la variable "sociedad transnacional" en la evolución de ambos procesos de integración. Finalmente, se procede a examinar interacciones entre actores no estatales $y$ procesos de integración, con el objetivo de determinar las motivaciones que fundamentan tales intercambios, así como, sus principales efectos.

\section{LA INTEGRACIÓN REGIONAL COMPARADA: LA NECESIDAD DE UN MARCO TEÓRICO}

Las escasas iniciativas comparativas existentes hasta la fecha, han sido realizadas desde la perspectiva del "neo-regionalismo" (Söderbaum y Shaw, 2003) que supone una "estructuración regional del mundo". Según este enfoque macro-estructural, la comparación se limita al uso de variables típicas de los estudios en relaciones internacionales (nivel de regionalización, nivel de integración económica) o de la economía política internacional (Mattli, 1999), dejando de lado la dimensión sociológica de la integración (Saurugger, 2008). Desde nuestra perspectiva, la integración regional se define como un "proceso mediante el cual emergen, se organizan y se estabilizan vínculos horizontales $y$ verticales entre actores políticos, económicos y sociales para la 
implementación de reglas del juego y procesos" (Fligstein y Stone, 2001).

Con el objetivo de determinar la relevancia de la sociedad transnacional para los procesos de integración regional, este artículo se inspira en la teoría neo institucionalista propuesta por Sweet y Sandholtz (Stone; Sandholtz y Fligstein, 2000: 12). Este modelo permite analizar la acción pública a través de actores múltiples que interactúan con diversos recursos, oportunidades y limitaciones institucionales. La evolución del sistema de integración se explica a través de la interacción de tres variables: las organizaciones supranacionales, las reglas supranacionales $y$ la sociedad transnacional. Según estos autores, la interacción de estas tres variables explica la evolución del proceso de integración: "for some of the dynamics of integration, namely, that movement in any one of the dimensions will tend to produce movement in the other two" (Stone; Sandholtz $y$ Fligstein, 2000: 12). Se trata de un sistema de retroalimentación entre la expansión de la sociedad transnacional y la institucionalización de la integración regional.

\subsection{LA SOCIEDAD TRANSNACIONAL, UN ACTOR CADA VEZ MAS RELEVANTE}

La noción de "sociedad transnacional" puede parecer ambigua: ¿quién participa efectivamente en el proceso? Esta noción encuentra sus raíces en la transaction-based theory de Sweet y Sandholtz y en la concepción de social exchange among individuals propuesta por Deutsch (1996). Según estos autores, el aumento de los intercambios sociales favorece la emergencia de demandas de regulación a nivel transnacional. La sociedad transnacional se encuentra compuesta de:

... actores no gubernamentales que participan en intercambios intra-europeos (sociales, económicos, políticos) y por consiguiente, inciden de manera directa $o$ indirecta en el proceso de toma de decisiones $y$ en los resultados políticos a nivel europeo (Sweet y Sandholtz, 1998: 12) ${ }^{1}$.

$1 \quad$ Traducción del autor.
Según numerosos estudios académicos sobre el activismo transnacional, la sociedad civil transnacional se ha convertido en un actor transnacional y/o global cada vez más relevante (Keck y Sikkink, 1998; Tarrow, 1998; Della Porta y Tarrow, 2004). Tales estudios la afirman como la transnational society matters. Al respecto, ponen de relieve dinámicas de interés, tales como: el "efecto boomerang" y la contestación social.

\subsection{EL ANÁLISIS DE LA INTERACCIÓN ENTRE ACTORES NO ESTATALES E INSTITUCIONES REGIONALES}

Una vez que el proceso de integración regional se encuentra muy avanzado y que los actores sociales se han articulado a nivel regional, la cuestión principal se trata más bien, de analizar las modalidades concretas de interacción entre los actores no estatales y las instituciones regionales. ¿Cuál es la función de los actores no estatales a nivel regional?, ¿ejercen estos algún tipo de influencia sobre las políticas públicas? Al mismo tiempo, las instituciones regionales también pueden modelar las estrategias y las formas organizativas de los actores no estatales.

En el marco de los estudios sobre el proceso de integración europeo, este tipo de cuestionamientos han sido planteados en torno a la noción de "europeanización". Este concepto se refiere a los procesos de ajuste o de adaptación al proceso de integración europea (Graziano y Vink, 2007 y EPPIE en Palier; Surel et ál, 2007). La principal dificultad de este tipo de análisis consiste en el énfasis puesto en el estudio de la interacción. El sistema político regional se considera, al mismo tiempo, como variable dependiente $y$ variable independiente, por lo cual, la frontera entre las causas y los efectos no es siempre evidente (Bulmer y Radaelli, 2004). Se trata por tanto de un prisma de análisis reciente y estimulante, pero, al mismo tiempo, difícil de implementar (EPPIE en Palier; Surel et ál, 2007). Por tanto, cuando se trata de analizar las interacciones entre la sociedad transnacional ${ }^{2}$ y un proceso de integración

2 Se tomó en cuenta la definición de Sweet y Sandholtz que caracteriza la sociedad transna- 
regional se deben tomar en cuenta dos dimensiones: el impacto del sistema regional sobre la sociedad transnacional y el impacto de esta sobre el sistema regional. El estudio simultáneo de estas dos dimensiones permite integrar en el análisis el estudio de feedback loops.

Aunque a menudo no existan obligaciones legales, los sistemas regionales ofrecen oportunidades a los actores no estatales, trátese de oportunidades de diálogo, de influencia o económicas (Sánchez, 2007). Es cierto que los actores que componen la sociedad transnacional deciden libremente utilizar o no tales oportunidades. Sin embargo, una vez que se decide utilizarlas, se asiste a un proceso que tiene consecuencias, tanto para la propia estructura de la sociedad transnacional, como para el proceso de integración regional.

La principal dificultad metodológica se trata de la construcción de la comparación, puesto que implica regiones muy diferentes. El proceso de integración europea es uno de los más avanzados. Al contrario, el proceso de integración centroamericano, a pesar de una larga herencia histórico-cultural, se caracteriza por la debilidad institucional y por la inestabilidad política. Teniendo en cuenta que siempre es posible cuestionar cualquier tipo de comparación (por ser los casos demasiado similares o demasiado diferentes), se considera que lo más relevante no es preguntarse si es posible comparar o si se debe comparar, sino más bien cómo comparar (Hassenteufel, 2005). Según esta perspectiva, la atención no se dirige tanto a la comparabilidad de los casos, sino más bien a la construcción de un marco teórico, que teniendo en cuenta las diferencias contextuales, permite la comparación. Tanto en Europa como en Centroamérica es posible identificar un sistema institucional a nivel regional $y$ una sociedad transnacional en interacción con este sistema.

cional como those nongovernmental actors who engage in intra-EC exchanges - social, economic, political - and thereby influence, directly or indirectly policymaking processes and outcomes at the European level (1998: 12).
Los elementos empíricos de este artículo provienen de dos estudios sobre las interacciones entre actores no estatales y el sistema político regional. El estudio sobre el caso europeo se centra en el análisis del impacto de la Unión Europea sobre la sociedad civil (Sánchez, 2007); mientras que el estudio sobre el caso centroamericano trata acerca de la incidencia de los actores no estatales sobre el proceso de integración regional (Parthenay, 2009). En ambos casos, la información proviene de documentación primaria y secundaria, así como, de entrevistas semi-directivas.

\section{LA RELEVANCIA DE LOS ACTORES NO ESTATALES EN LA EVOLUCIÓN DE LOS PROCESOS DE INTEGRACIÓN EUROPEO Y CENTROAMERICANO}

Según Sweet y Sandholtz (2000), el grado de desarrollo de una sociedad transnacional constituye un indicador del avance del proceso de integración regional. Las reglas e instituciones regionales pueden contribuir a la emergencia de una sociedad transnacional $y$ al mismo tiempo, la conformación de una sociedad transnacional puede contribuir al desarrollo del proceso de integración. En esta sección, se analiza la trayectoria socio-histórica de las interacciones entre los procesos de integración europeo y centroamericano. Tal y como habían previsto Sweet y Sandholz, se destaca una correlación entre el nivel de competencias del sistema político regional y el número de actores no estatales y grupos activos a este nivel (Mazey y Richardson, 2001).

Antes de proceder al análisis, es preciso señalar que en Centroamérica no existen instituciones supranacionales comparables a las existentes en Europa. Por tanto, la sociedad transnacional ha contribuido más al fortalecimiento de las instituciones regionales. En este sentido, se puede considerar que en el caso centroamericano, el proceso de integración responde a lógicas bottom-up. Sin embargo, si los actores transnacionales en Centroamérica disponen de recursos necesarios para poder fortalecer el proceso de integración, es gracias a 
los fondos de la cooperación internacional, que procede en gran parte de la Unión Europea (UE). Los fondos europeos (y norteamericanos) han contribuido así, a promover el fortalecimiento de la sociedad transnacional, tanto en Europa como en Centroamérica, lo que supone un proceso top-down a nivel europeo y un proceso que puede parecer bottom-up en el nivel centroamericano.

\subsection{INTEGRACIÓN REGIONAL Y SOCIEDAD TRANSNACIONAL DÉBIL (1950-1990)}

En un primer momento, las comunidades europeas y la integración centroamericana no disponían de un gran número de competencias, ni tampoco de la capacidad necesaria para imponer decisiones a los estados miembros en la mayoría de los ámbitos, lo que se tradujo en un desarrollo muy débil de una sociedad transnacional.

CUADRO 1

LOS INSTRUMENTOS JURÍDICOS DE INTEGRACIÓN REGIONAL

\begin{tabular}{|c|c|c|}
\hline & AMÉRICA CENTRAL & UNIÓN EUROPEA \\
\hline Principales tratados & $\begin{array}{l}\text { Organización de los Estados Centroamericanos - } \\
\text { ODECA (1951) } \\
\text { Protocolo de Tegucigalpa (1991) } \\
\text { Protocolo de Guatemala (1993) } \\
\text { Alianza para el Desarrollo Sostenible de Centroamérica } \\
\text { - ALIDES (1994) }\end{array}$ & $\begin{array}{l}\text { Tratados de Roma (1957) } \\
\text { Acta Única Europea (1988) } \\
\text { Tratado de Maastricht (1993) } \\
\text { Tratado de Lisboa (2009) }\end{array}$ \\
\hline $\begin{array}{l}\text { Sociedad transnacional } \\
\text { Años } 1950\end{array}$ & $\begin{array}{l}\text { Cámaras de comercio : } \\
\text { Federación de entidades privadas de Centroamérica, } \\
\text { Panamá y República Dominicana - FEDEPRICAP } \\
\text { (1950) } \\
\text { Federación de cámaras y asociaciones industriales } \\
\text { centroamericanas y de la República Dominicana - } \\
\text { FECAICA (1959) } \\
\text { Federación de cámaras de comercio de Centroamérica } \\
\text { - FECAMCO (1961) } \\
\text { Sindicatos : } \\
\text { Federación de cámaras de comercio de Centroamérica } \\
\text { - CCT (1968) }\end{array}$ & $\begin{array}{l}\text { Empresas: } \\
\text { Unión de las industrias de la } \\
\text { Comunidad europea - UNICE } \\
\text { (1958) } \\
\text { Agricultura: } \\
\text { Comité de organizaciones } \\
\text { profesionales agrícola - COPA } \\
\text { (1958) } \\
\text { Confederación General de } \\
\text { Cooperativa Agrarias en la Unión } \\
\text { Europea - COCEGA (1959) }\end{array}$ \\
\hline $\begin{array}{l}\text { Sociedad transnacional } \\
\text { Años } 70 \text { y } 80\end{array}$ & $\begin{array}{l}\text { Derechos Humanos: } \\
\text { Fundación para la defensa de los Derechos Humanos - } \\
\text { FUNDEHUCA (1978) } \\
\text { Académico : } \\
\text { Asociación de las universidades privadas de } \\
\text { Centroamerica y Panamá - AUPRICA (1985) } \\
\text { Servicio: } \\
\text { Confederación de Cooperativas del Caribe, Centro y } \\
\text { Suramérica - CCC-CA (1980) }\end{array}$ & $\begin{array}{l}\text { Intereses no comerciales: } \\
\text { Cooperación al desarrollo: } \\
\text { CLONG (1978) } \\
\text { Medio ambiente: } \\
\text { Oficina Europea de Medio } \\
\text { Ambiente - BEE }\end{array}$ \\
\hline $\begin{array}{l}\text { Sociedad transnacional } \\
\text { Años } 1990 \text { y } 2000\end{array}$ & $\begin{array}{l}1990 \text { : se estiman } 15 \text { grupos presentes a nivel } \\
\text { centroamericano } \\
2000 \text { : se estima más de } 70 \text { grupos activos al nivel } \\
\text { regional }\end{array}$ & $\begin{array}{l}\text { 1990: se estiman } 3000 \text { grupos } \\
\text { presentes a nivel europeo } \\
2000 \text { : se estiman entre } 15000 \mathrm{y} \\
20000 \text { grupos a nivel europeo }\end{array}$ \\
\hline
\end{tabular}

Fuente: Elaboración propia. 
Los primeros grupos de interés activos a nivel regional trabajaban en el ámbito de las competencias de las instituciones regionales. En el caso de las comunidades europeas, los grupos europeos se crean de manera paralela al establecimiento de nuevas competencias en un determinado sector (Sidjanski, 1997). En un primer momento del proceso de integración, los únicos grupos que se estructuran a nivel europeo son los grupos empresariales, asociaciones de consumidores y organizaciones agrícolas. Algunos autores afirman que el proceso de integración europeo precede a la emergencia de los actores no estatales a nivel europeo (Sidjanski, 1997). En efecto, durante este periodo, la acción de instituciones supranacionales $y$ en particular, el activismo de la Comisión europea contribuyó a la orientación europea, es decir, a la creación de un determinado número de actores sociales, principalmente ongs (Cram, 1997; Harvey, 1993; Greenwood, 2003; Sánchez, 2007). Esta dinámica también se aplica a grupos que reúnen actores económicos o empresas. La Mesa europea de industriales (European Round Table-ERT) fue creada con el apoyo de Etienne Davignon, comisario de la Dirección General Industria de la Comisión Europea (Green en Greenwood, 2004 y Greenwood, 2003). Durante este periodo, se asiste a una conjunción de intereses entre las instituciones y los actores supranacionales.

En Centroamérica, desde el nacimiento de la ODECA (1951), las interacciones entre actores no estatales e instituciones de integración son escasas debido a la ausencia de instituciones supranacionales $y$ a la debilidad de la institucionalidad regional. El proceso de integración también se centró en un primer momento, en el sector económico (Mercado Común Centroamericano, proyecto de la Unión Aduanera Centroamericana). Del total de organizaciones existentes, cuatro pertenecen al sector privado, tres al sector sindical y una al sector de los derechos humanos. El sector empresarial es mayoritario y el discurso de los sindicatos se centra en objetivos de naturaleza económica. Al respecto, es preciso señalar que la sociedad transnacional enfrenta una difícil estructuración de sus intereses por razones ideológicas, políticas y/o materiales (Grinspun; Alvarenga y Shamsie, 1998).

Durante este periodo, únicamente los actores del sector económico se movilizaron en el espacio regional. Los grupos representativos de este sector jugaron un papel crucial en la dinamización de los intercambios económicos $y$ aportaron un apoyo substancial a las instituciones regionales en materia de competencias técnicas (Dabène, 2009). El proyecto de creación de la Comunidad Económica y Social Centroamericana (CESCA) permitió la participación de los actores económicos dentro de un comité de alto nivel para la reestructuración del Mercado Común Centroamericano (MCCA).

También, en este caso es posible afirmar que existe una correlación entre el nivel de desarrollo de las instituciones regionales $y$ de la sociedad transnacional. Sin embargo, las instituciones centroamericanas no contribuyeron directamente a la conformación de la sociedad transnacional, tal y como sucedió en el caso europeo. En esta época, el marco institucional era demasiado débil, ya que no existía ninguna institución supranacional con poder de iniciativa, como es el caso de la Comisión Europea.

\subsection{DESARROLLO DEL PROCESO DE INTEGRACIÓN Y DE LA SOCIEDAD TRANSNACIONAL (1900) Y LOS PRIMEROS INTENTOS DE REGULACIÓN}

A principios de los años 90, se destaca una aceleración del proceso de integración regional, tanto en Europa como en Centroamérica. Se asiste a una expansión de las competencias institucionales en ambos procesos de integración, lo que implicó el desarrollo de la sociedad transnacional. En este periodo, es preciso resaltar los primeros intentos de regulación/institucionalización.

A finales de los años 80, con la firma del Acta Única Europea y posteriormente, del Tratado de Maastricht, un gran número de actores no estatales se movilizan a nivel europeo (Fligstein y Stone, 2001). Este periodo se caracteriza por la emergencia de grupos que defienden el interés general. Tal desarrollo se encuentra vinculado a la extensión de las competencias del sistema de integración europeo y a la extensión del sistema de votación por 
mayoría calificada. Una transferencia de poder a nivel europeo, crea las condiciones necesarias para el desarrollo de grupos a este nivel (Aspinwall y Greenwood, 1998). A principios de los años 90, se contabilizan alrededor de 3000 grupos activos a nivel europeo.

El proceso de democratización y de apertura de las instituciones regionales en Centroamérica, también coincidió con la emergencia de numerosos grupos y asociaciones a nivel regional. A finales de la década de 1980, se asiste a una ola de democratización y al renacimiento del proceso de integración en esta región. Con la pacificación, se ratifican dos tratados, el Protocolo de Tegucigalpa (1991) $y$ el Protocolo de Guatemala (1993). El nuevo Sistema de Integración Centroamericano (SICA) dispone de más competencias institucionales, lo que supone una ampliación sectorial de la integración. El SICA se abre al sector social, ambiental o al sector servicios. En 1998, alrededor de 40 organizaciones regionales cuentan con existencia jurídica. La emergencia de esa nueva sociedad transnacional en la década de 1990 concuerda con la ampliación sectorial del SICA. La inclusión del sector académico, del sector ambiental, del sector étnico-poblacional, contribuye a la emergencia de nuevos grupos a nivel regional.

Durante este periodo, se asiste también a los primeros intentos de regulación de la participación de la sociedad transnacional en los procesos de toma de decisiones. En Europa, la Comisión Europea en respuesta al fuerte incremento de las actividades de lobbying a nivel europeo, publica una comunicación sobre este tema $y$ un repertorio de grupos de interés a principios de los años 90 (Comisión Europea, 2001 y 1993). El Parlamento Europeo aprueba los primeros informes sobre las actividades de los grupos de interés, lo que da lugar a un código de conducta obligatorio aplicable a partir de 1997 (Vayssière, 2002). En Centroamérica, varias iniciativas por parte de la sociedad civil se desarrollan en el marco del SICA, tales como, la Iniciativa Civil para la Integración Centroamericana (ICIC) o el Consejo Centroamericano de Coordinación Intersectorial (CACI). Ambas coaliciones de organizaciones regionales se crearon con el propósito de representar amplios sectores y de debatir asuntos de carácter regional, para tratar de influir en el proceso de integración (Grinspun; Alvarenga y Shamsie, 1998). En 1997, el SICA y la cooperación internacional también desarrollaron algunas iniciativas para promover la participación de la sociedad transnacional. Un seminario regional, titulado "Participación de la sociedad civil en la integración centroamericana" en San Salvador y la publicación "Hacia una integración desde abajo", resaltan la necesidad de participación social en el SICA.

\subsection{INSTITUCIONALIZACIÓN DE LA SOCIEDAD TRANSNACIONAL (1998-2010)}

A finales de los años 90 se asiste a una crisis de legitimidad en ambos procesos de integración, lo que lleva al establecimiento de un nuevo tipo de institucionalización de las relaciones entre las instituciones regionales y la sociedad transnacional.

El número de grupos activos a nivel europeo ha seguido aumentando durante la década del 2000, lo que hace que actualmente se estime que el número de grupos se encuentra entre 15000 y 20000 (Cohen y Richardson, 2009). El fin de los años 90, supone un cambio significativo del tipo de relaciones entre las instituciones europeas $y$ la sociedad transnacional. En este momento, la sociedad transnacional (o la sociedad civil si se retoma el vocabulario de las instituciones europeas) pasa de ser un aliado en el proceso de integración a un instrumento de legitimación (Sánchez, 2007). Este cambio de percepción encuentra su origen en dos fenómenos principales. En primer lugar, el proceso de integración europeo se ve confrontado a una crisis de legitimidad y se precisa encontrar soluciones a este problema. Esta crisis se manifiesta en la Comisión Europea con la dimisión de la Comisión Santer en 1999 y a nivel del Consejo de Ministros y del Consejo Europeo, en la dificultad por ratificar los tratados europeos. La crisis de legitimidad adquiere su máxima expresión durante el rechazo del Tratado Constitucional en Francia y Holanda. 
Esta nueva concepción del papel de la sociedad transnacional se traduce en la creación de mecanismos de participación, a partir del año 2000. El libro blanco sobre la gobernanza europea, parte de la constatación de que los ciudadanos europeos se encuentran cada vez más alejados de la Unión. Entre las soluciones propuestas, se destaca la participación de la sociedad civil. A este efecto, el secretariado general de la Comisión Europea de Romano Prodi, instaura un proceso de consulta de la sociedad civil en el seno de todos sus servicios (Comisión Europea, 2002). Del mismo modo, otras instituciones europeas como el Comité Económico y Social e incluso algunas formaciones del Consejo de Ministros, empiezan a promover el diálogo con la sociedad civil. El diálogo con la sociedad civil se integra también en las nuevas formas de gobernanza, como el Método Abierto de Coordinación (MOC) o en el marco de las convenciones creadas para la redacción de la Carta de Derechos Fundamentales o del Tratado Constitucional. A pesar de una participación activa de la sociedad civil, el modelo de participación que finalmente se adopta responde más a las necesidades institucionales que a las aspiraciones de la sociedad civil.

En Centroamérica, el renacimiento de la institucionalidad regional a principios de la década de los 90, no produjo los resultados esperados. A finales de la década, el Sistema de Integración afronta una crisis institucional $y$ por consiguiente, una crisis de legitimidad. La mayoría de los órganos del SICA solo tienen existencia formal. Las instituciones regionales no reciben suficiente financiamiento por parte de los estados miembros (\$20 000) y los resultados son escasos. La reforma del Comité Consultivo del SICA (CC-SICA) en 1996, después de cuatro años de existencia formal sin realizaciones concretas, constituye una manifestación de esta tendencia. El Protocolo de Tegucigalpa tiene valor jurídico, pero no dispone de los recursos ni de la capacidad administrativa necesarios para garantizar su funcionamiento (Caldentey y Romero, 2010). Este fenómeno se aplica en mayor o menor medida al conjunto de las instituciones de integración regional, lo que ha llevado al cuestionamiento de la legitimidad de las instituciones del SICA.

La sociedad transnacional también se convierte en un instrumento estratégico utilizado por las instituciones regionales para legitimar la reconfiguración del marco institucional de la integración. Las reformas actuales están marcadas por la voluntad de democratización del proceso de integración regional. Si la creación de mecanismos de participación precede a las reformas institucionales, la sociedad civil representa un factor democratizador de la institucionalidad regional. La inclusión de la sociedad civil responde a las exigencias de buen gobierno, una preocupación presente en el ámbito de la cooperación internacional, especialmente en el Programa de Apoyo a la Integración Regional Centroamericana (PAIRCA, 1999). También deben tenerse en cuenta los esfuerzos de la sociedad civil centroamericana para adaptarse a las exigencias de los donantes internacionales con el fin de captar fondos.

Como en el caso europeo, la nueva concepción de la sociedad transnacional se traduce por la institucionalización de mecanismos formales de cooperación entre las instituciones del SICA y la sociedad civil. La participación de la sociedad civil constituye un tema tratado en cada una de las Cumbres de Jefe de Estado, órgano supremo del SICA. Además, una nueva reforma radical del CC-SICA tiene lugar en 1999, con el apoyo técnico de una organización de la sociedad civil: Fundación para la Paz y la Democracia (FUNPADEM). El Plan Estratégico del CC-SICA fue presentado en 2002, ante los presidentes reunidos en ocasión de la XXII Cumbre de Jefes de Estado.

Se destaca igualmente, la creación de nuevos mecanismos formales de colaboración con la sociedad civil. Los órganos de consulta de la sociedad transnacional se expanden a otros sectores, como el sector ambiental (Foro Social Centroamericano de Ambiente y Desarrollo FOSCAD), económico (Comité Consultivo de la Integración Económica - CCIE) y social (Comité Consultivo de Integración Social - CCIS). Esta nueva interpretación del papel de la sociedad civil se refleja también en la creación 
de mecanismos ad hoc de colaboración técnica (Memorandum de Entendimiento) o de financiamiento, con fondos específicos de la Unión Internacional para la Conservación de la Naturaleza (UICN) sobre ambiente o con foros de donantes en la Comisión Centroamericana de Ambiente y Desarrollo (CCAD). A finales de la primera década del 2000 , la institucionalización de la sociedad transnacional ha modificado radicalmente los modos de regulación y de gobernabilidad en la región centroamericana.

La tercera parte de este artículo introduce una perspectiva sociológica; se trata de resaltar la manera en que los actores sociales utilizan de manera estratégica los mecanismos de participación y de consulta regionales. Al mismo tiempo, los actores sociales también pueden ser instrumentalizados por las instituciones regionales.

\section{LA CONSULTA DE LOS ACTORES NO ESTATALES: ¿EXIGENCIA POLÍTICA O NECESIDAD TÉCNICA?}

Tal y como se desprende de la sección anterior, los procesos de integración centroamericano y europeo han desarrollado mecanismos de consulta de los actores no estatales. En ambos casos, es posible establecer una distinción conceptual entre las consultas orientadas hacia la adquisición de competencias técnicas, que permiten obtener mejores resultados en materia de políticas públicas (output legitimacy) y las consultas generales de carácter político, creadas para garantizar la participación de la sociedad civil (input legitimacy). Al respecto, las relaciones entre sociedad civil e instituciones regionales pueden ser abordadas tanto desde la perspectiva neofuncionalista como desde la intergubernamentalista. En efecto, el establecimiento de relaciones con los actores de la sociedad civil puede responder a necesidades técnicas, a una voluntad de expansión de competencias o de legitimación de las instituciones supranacionales (perspectiva neo-funcionalista), pero también a preferencias estatales. El origen de la dinámica de participación afecta la forma en que se forman las prácticas y el grado de institucionalización de las mismas.

\subsection{LOS LÍMITES DE LOS MECANISMOS FORMALES} DE PARTICIPACIÓN: FALTA DE EFECTIVIDAD

A nivel europeo (con la excepción del Comité Económico y Social), los tratados no confieren ningún derecho de participación a las organizaciones de la sociedad civil. La mayoría de prácticas de participación, desarrolladas por las instituciones supranacionales, son abiertas e informales $y$ han sido, solo parcialmente plasmadas en el más reciente de los tratados. Sin embargo, a nivel centroamericano, los procedimientos de participación de la sociedad civil resultan de una decisión adoptada por los jefes de gobierno $y$ se encuentran directamente previstos, en los tratados desde el principio de los años 90. Esta sección mostrará, que aunque la Unión Europea promueve prácticas formales de diálogo con la sociedad civil en terceros países, tales prácticas han sido solamente integradas en los tratados europeos recientemente $y$ de manera informal.

En el caso del proceso de integración europeo, en un primer momento, la participación formal de los actores no estatales se limitó en efecto al marco estricto del Comité Económico y Social Europeo (CESE), órgano consultivo creado en 1957, típico de las organizaciones internacionales creadas durante este periodo e inspirado en el modelo corporatista de representación de intereses. La peculiaridad de esta instancia (conformada por tres grupos: empresarios, trabajadores y actividades diversas) es que sus 344 miembros son nombrados por los estados miembros, lo que supone que las asociaciones regionales europeas no se encuentran representadas. A pesar de garantizar un cierto tipo de participación, no es posible afirmar que el CESE haya contribuido al desarrollo de una sociedad transnacional a nivel europeo, ya que únicamente se garantiza la participación de grupos nacionales.

Se destaca también el poder restringido de esta institución, que se limita a emitir dictámenes sobre las propuestas legislativas. Esta situación explica el por qué la mayoría de actores no estatales no demuestran demasiado interés por el CESE. Como señala un representante de Amnesty International: "en este contexto, la sociedad civil ha sabido desenvolverse muy 
bien con las instituciones (europeas) sin que el apoyo del CESE halla sido necesario".

A parte del CESE, los tratados europeos no crean ningún mecanismo formal de consul$\mathrm{ta}^{3}$. A pesar de una gran insistencia de diversos actores no estatales, no se prevé una base jurídica para la participación de la sociedad civil en la UE hasta el Tratado de Lisboa de 2009. En su artículo 8B este tratado afirma que las instituciones europeas "mantendrán un diálogo abierto, transparente y regular con las asociaciones representativas y la sociedad civil". Se debe resaltar el carácter extremadamente abierto de esta disposición. No existe ninguna obligación concreta para las instituciones europeas ni se requiere ningún tipo de condición para participar en este tipo de diálogo. Este mismo tipo de concepción se desprende de los procesos de consulta propuestos desde principios del año 2000, por el secretariado general de la Comisión Europea. Aunque se definen unas normas mínimas que la Comisión se propone respetar (Comisión Europea, 2002), estos procesos de consulta no confieren ningún derecho concreto a los ciudadanos europeos, en tanto que este tipo de consulta no está prevista por ninguna norma comunitaria. Se trata simplemente de una propuesta planteada en una comunicación de la Comisión. Estos procesos de consulta se dirigen a todas las "partes interesadas" sin ningún tipo de discriminación. Cualquier tipo de asociación o individuo puede participar en el proceso. No existe ningún tipo de exigencia en materia de personalidad jurídica, de representatividad, de nacionalidad $o$ de estatuto. Por tanto se puede destacar que el sistema de representación de los intereses existente a nivel europeo desarrollado durante la década del 2000, tiende hacia el pluralismo.

Aunque estos procesos de consulta hayan sido creados para reducir el déficit democrático y crear vínculos con los ciudadanos de la Unión Europea, el sistema de consulta actual ha sido el objeto de numerosas críticas. En mayoría de

3 Los tratados sucesivos ni siquiera mencionan a la sociedad civil o actores no estatales, si se exceptúan dos declaraciones anexas a los tratados, durante los años 90, sobre los grupos religiosos y la política social. estudios sobre los nuevos procedimientos de consulta, se desprende que los grupos que disponen de más recursos participan mucho más en las consultas (Persson, 2007). También se resalta que el tipo de feedback o de influencia directa en el proceso político europeo es muy limitado. Del mismo modo, se ha criticado la ausencia de criterios para determinar quien participa en los procesos de consulta $y$ que contribuciones son tomadas en cuenta (Fazi y Smith, 2006; Quittkat y Finke, 2008).

Durante años, los mecanismos oficiales de consulta regional en Centroamérica no tuvieron efectividad concreta sea por falta de recursos o de voluntad política. Por esta razón, tales espacios han sido a menudo calificados como "espacios vacios". Los procedimientos de consulta empiezan a materializarse solo recientemente. La consolidación institucional de los procesos de consulta oficial es el producto de una evolución radical del uso estratégico (recursos financieros, competencias técnicas) $y$ de la instrumentalización, por parte de las instituciones regionales con la perspectiva de democratización de la institucionalidad regional (CEPAL-BID, 1997; Grinspun, Alvarenga y Shamsie, 1998). El trabajo institucional resulta también de una estrecha colaboración e intercambios de experiencia con el CESE europeo (Carrillo, 2006; 2008).

Los procedimientos de participación de la sociedad transnacional en el CC-SICA $y$ el CCIE son muy limitados $y$ privilegian a las organizaciones con recursos. El CC-SICA (Estatuto, 2007: 10) y el CCIE (Reglamento Interno, 2006: 5) ${ }^{4}$ se componen de "organizaciones regionales representativas de la sociedad civil comprometidas en el esfuerzo de integración ístmica" (art.4). Para ser miembro se deben respetar criterios de regionalidad en términos de estatus formal de la organización (personalidad jurídica), de sede y de naturaleza de las actividades. También, hay que "representar a un significativo número de entidades nacionales y que éstas representen a un considerable número de individuos" (art.4). En el caso del CC-SICA, las organizaciones miembro deben pagar cuotas anuales para participar.

4 Resolución nro. 140-2005 (COMIECO-EX). 
A pesar de la inclusión de nuevos actores en la integración, por el momento no se ha concretizado una influencia efectiva sobre el contenido de las políticas en el sistema regional. Los debates y las propuestas formuladas por la sociedad civil, en espacios como el CC-SICA, el CCIE o el FOSCAD se limitan a ser espacios de debate sobre la integración regional. Los intereses de los grupos organizados no son finalmente tomados en cuenta, debido a las dificultades de implementación de los programas o proyectos.

\subsection{LA CONSULTA TÉCNICA: ¿INFLUENCIA O INSTRUMENTALIZACIÓN?}

Las instituciones regionales también han desarrollado mecanismos de consulta informales con los actores no estatales. Tales mecanismos preceden a menudo a los mecanismos formales de participación y parecen responder a la necesidad de las instituciones regionales de contar con las competencias técnicas de los actores no estatales, para poder definir mejor las políticas públicas. Los actores no estatales también permiten a las instituciones obtener informaciones previas sobre los posibles impactos de las políticas públicas y sobre el grado de rechazo de las mismas (Greenwood, 2003). En muchos casos, los actores no estatales se hacen cargo de tareas administrativas (Saurugger, 2002).

En el caso de la integración europea, varias direcciones generales han desarrollado vínculos muy importantes con los actores no estatales según sus intereses específicos. La dirección general de agricultura ha establecido un gran número de comités técnicos, donde participan las principales organizaciones agrícolas europeas. También se trata del caso de las direcciones generales sobre medio ambiente, empresas, asuntos sociales, desarrollo, entre otras. Cabe resaltar que las cuestiones técnicas no se limitan a asuntos relacionados con la low politics, ya que también pueden referirse a temáticas normalmente consideradas high politics como la diplomacia. En efecto, las principales organizaciones de derechos humanos activas a nivel europeo son a menudo consultadas por la Comisión Europea, acerca de su posición sobre la situación de derechos humanos en un determinado país. Este tipo de información detallada es utilizada por los funcionarios europeos para preparar el diálogo político con determinados países. Se puede considerar como información "técnica" maciones sobre casos concretos de violaciones de derechos humanos.

A finales de los años 80, el SICA también solicita la contribución de numerosos actores sociales. Empieza un periodo de estrecha colaboración a nivel técnico. El sector ambiental y comercial son casos paradigmáticos del uso estratégico de la sociedad transnacional. Los actores sociales apoyaron la creación institucional del subsistema ambiental centroamericano $y$ pusieron a la disposición del sistema institucional regional, sus competencias técnicas, así como, sus recursos financieros u organizativos. Esta estrategia de externalización de la acción regional compensaba la falta de recursos económicos y la debilidad de los presupuestos consagrados a la integración regional por los Estados de la región. Por tanto, se puede afirmar que una parte de la acción pública regional fue externalizada.

En el ámbito comercial, la consulta se hace a nivel técnico mediante estructuras ad hoc que se activan durante las negociaciones regionales. Estas consultas tienen lugar a través de los "Cuartos Adjuntos" o "Cuartos de lado", antes y durante la negociación y se suman a los mecanismos nacionales establecidos por la ley. Antes de la negociación, las cámaras cuentan con un espacio determinado para incidir en la elaboración del acuerdo. Durante la negociación, los representantes de las cámaras empresariales, así como, de las propias entidades interesadas, pueden acompañar a la delegación gubernamental a las reuniones, de manera que existe un contacto e intercambio de información constante entre los funcionarios, los empresarios $y$ asociaciones gremiales.

La débil institucionalización de los mecanismos de consulta y la posición predominante de organizaciones con mayores recursos, limita el número de actores con capacidad de incidencia

$5 \quad$ Intervención de Gregoire Therry, delegado de la FIDH en Bruselas el 6 de mayo de 2010. 
en el Sistema de Integración Centroamericano. En efecto, los procedimientos de consulta son monopolizados por un número muy reducido de grupos.

Tal y como se menciona previamente, este fenómeno se reproduce en el caso europeo. Los grupos que participan en los procesos de consulta europeos son aquellos que disponen de los recursos necesarios para organizarse a nivel europeo y responder a las demandas técnicas de las instituciones europeas. Sin embargo, en Europa existen organizaciones "expertas" en muchos ámbitos y no solo, en el ámbito de las políticas comerciales.

\section{CONCLUSIÓN: COMPARANDO EL PAPEL DE LOS ACTORES NO ESTATALES EN LOS PROCESOS DE INTEGRACIÓN REGIONAL}

Este estudio muestra que las variables destacadas por Stone Sweet et ál., contribuyen a la comprensión de la evolución de los procesos de integración. Efectivamente, tal y como estos autores proponen "movement in one of the dimensions will tend to produce movement in the other two", propuesta que queda cercana de la hipótesis del spill-over conceptualizado por Schmitter (1970). El regionalismo comparado también muestra que las variables más relevantes no son siempre idénticas en cada uno de los procesos de integración regional. En el caso europeo, la existencia de instituciones supranacionales contribuye a la emergencia y desarrollo de una sociedad transnacional. En algunos casos menos frecuentes, los actores nacionales y transnacionales también contribuyen a la emergencia de nuevas normas.

El análisis de la interacción entre los grupos de interés y los diferentes sistemas de integración regional plantean la cuestión de la calidad de las prácticas democráticas en dichos sistemas. En efecto, las instituciones regionales consideran que los organismos de la sociedad civil pueden constituir una fuente de legitimidad. Al respecto, seria pertinente dedicar más espacio al análisis de la contribución de estas prácticas al desarrollo de la democracia, en ambos procesos de integración regional. Entre las preguntas que se plantean en este estudio, se destaca el interés por el cambio institucional. En efecto, estudiar el impacto de las interacciones entre actores conduce a describir las relaciones y juegos de poder, ya se trate de sus consecuencias o de las configuraciones institucionales.

\section{BIBLIOGRAFÍA}

Aspinwall, Mark y Greenwood, Justin. Collective action in the European Union. London. Routledge, 1998: 238.

Bulmer, Simon y Radaelli, Claudio M. "The Europeanization of National Policy". Queen's Papers on Europeanization 1. 2004.

Caldentey del Pozo, José Juan y Romero Rodríguez, Pedro (eds.). El SICA y la UE: la integración regional en una perspectiva comparada. España: Ed. Córdoba. Fundación ETEA para el Desarrollo y la Cooperación, 2010.

Cohen, David y Richardson, Jeremy. Lobbying the European Union. Oxford: Oxford University Press, 2009.

Comisión Europea. Un dialogue ouvert et structuré entre la Commission européenne et les groupes d'intérêt $63 \mathrm{JO}$ C. 5 mars 1993.

Comisión Europea. Gouvernance européenne: un livre blanc 428 Final. COM, 25 juillet 2001.

Comisión Europea. Vers une culture renforcée de consultation et de dialogue. Principes généraux et normes minimales aux consultations engagées par la Commission avec les parties intéressées 704 Final. Bruxelles. CoM, 2002.

Cram, Laura. Policy-making in the European Union, conceptual lenses and the integration process. London. Routledge, 1997: 210.

Dabène, Olivier. The politics of Regional Integration in Latin America. Theoretical and comparative explorations. Palgrave McMillan, 2009.

Della Porta, Donatella y Tarrow, Sidney. Transnational activism. Cambridge University Press, 2004.

Deutsch, Karl. Integración y formación de comunidades políticas: análisis 
sociológico de experiencias históricas. Buenos Aires: BID-INTAL, 1996.

EPPIE. "Introduction. Analyser l'européanisation des politiques publiques". En: Palier, Bruno; Surel, Yves et ál. L'Europe en action: l'européanisation dans une perspective comparée. Paris. L'Harmattan, 2007: 13-86.

Fligstein, Neil y Stone Sweet, Alec. "Institutionalizing the Treaty of Rome". Stone Sweet, Alec; Fligstein, Neil y Sandholz, Wayne (eds.). The Institutionalization of Europe. Oxford. Oxford University Press, 2001: 29-55.

Graziano, Paolo y Vink, Maarten P. Europeanization. New Research Agenda. Houndmills: Palgrave, 2007.

Green Cowles, Maria. "Large firms and the transformation of $\mathrm{EU}$ Business Association: a historical perspective". Greenwood, Justin (ed.). The effectiveness of EU Business Associations. Houndmills: Palgrave, 2004.

Greenwood, Justin. Interest representation in the European Union. London. Macmillan, 2003: 310.

Grinspun, Ricardo; Alvarenga, Carlos y Shamsie, Yasmine. Hacia una integración desde abajo. Participación, Sociedad Civil e Integración Centroamericana. Proyecto SICA/CERLAC/ PAR, 1998.

Hassenteufel, Patrick. "De la comparaison internationale à la comparaison transnationale. Le déplacement de la construction d'objets comparatifs en matière de politiques publiques". Revue française de Science Politique 55 (1). 2005: 113-132.

Keck, Margaret y Sikkink, Katheryn. Activists beyond borders. Cornell University Press, 1998.

Mattli, Walter. The logic of regional integration: Europe and beyond. Cambridge: Cambridge University Press, 1999.
Mazey, Sonia y Richardson, Jeremy. "Institutionalizing promiscuity: Commission-Interest Groups relations in the EU". Stone Sweet, Alec; Sandholz, Wayne y Fligstein, Neil (eds.). The Institutionalization of Europe. Oxford. Oxford University Press, 2001: 71-93.

Parthenay, Kevin. "La régionalisation des mouvements sociaux en Amérique Centrale: le cas du CAFTA-RD”. Mémoire de DEA. Institut d'Etudes Politique de Paris, 2009.

Sánchez Salgado, Rosa. Comment l'Europe construit la société civile. Paris: Dalloz, 2007.

Saurugger, Sabine (ed.). "Les approches sociologiques de l'intégration européenne. Perspectives critiques". Politique européenne 25. 2008: 5-230.

Schmitter, Philippe C. "A revised Theory of Regional Integration". International Organization 24 (4). Regional Integration: Theory and Research. Autumn, 1970: 836-868.

Söderbaum, Frederik y Shaw, Thimothy M. Theories of new regionalism: a palgrave reader. Basingstoke: MacMillan, 2003.

Sidjanski, D. "Networks of European pressures groups. From the origins of the EEC to the EU". Euryopa articles \& Conferences 4. 1997.

Stone Sweet, Alec; Sandholtz, Wayne $y$ Fligstein, Neil (eds.). The Institutionalization of Europe. Oxford: Oxford University Press, 2000.

Tarrow, Sidney. Power in movement. Cambridge University Press, 1998.

Vayssière, Bertrand. Groupes de Pression en Europe. Toulouse: Privat, 2002.

Fecha de ingreso: 25/01/2011 Fecha de aprobación: 08/06/2011 
